\title{
Structural Similarity: Spectral Methods for Relaxed Blockmodeling
}

\author{
Ulrik Brandes \\ University of Konstanz, Germany \\ Jürgen Lerner \\ University of Konstanz, Germany
}

\begin{abstract}
In this paper we propose the concept of structural similarity as a relaxation of blockmodeling in social network analysis. Most previous approaches attempt to relax the constraints on partitions, for instance, that of being a structural or regular equivalence to being approximately structural or regular, respectively. In contrast, our approach is to relax the partitions themselves: structural similarities yield similarity values instead of equivalence or non-equivalence of actors, while strictly obeying the requirement made for exact regular equivalences. Structural similarities are based on a vector space interpretation and yield efficient spectral methods that, in a more restrictive manner, have been successfully applied to difficult combinatorial problems such as graph coloring. While traditional blockmodeling approaches have to rely on local search heuristics, our framework yields algorithms that are provably optimal for specific data-generation models. Furthermore, the stability of structural similarities can be well characterized making them suitable for the analysis of noisy or dynamically changing network data.
\end{abstract}

Keywords: Social network analysis; Blockmodeling; Spectral graph partitioning; Conflict networks; Dynamic network visualization.

We gratefully acknowledge financial support from DFG (grant $\mathrm{Br} 2158 / 2-3$ ) and University of Konstanz under grant FP 626/08.

Corresponding Author's Address: Jürgen Lerner, Department of Computer \& Information Science, University of Konstanz, Box 67, 78457 Konstanz, Germany, tel. +49 7531 88-4436, fax +49 7531 88-3577, e-mail: lerner@inf.uni-konstanz.de. 


\section{Introduction}

The notion of (structural) position is fundamental in social network analysis, see e. g., Wasserman and Faust (1994), Borgatti and Everett (1992). Actors are said to occupy the same position if they have similar patterns of ties to other actors and the task of determining such classes of actors is referred to as blockmodeling (Doreian, Batagelj, and Ferligoj 2005) or role assignment (Everett and Borgatti 1991).

Various types of role assignment differ in how they operationalize the notion of "similar patterns of ties to other actors." Concrete instances include structural equivalence (Lorrain and White 1971), regular equivalence (White and Reitz 1983), automorphic equivalence (Borgatti and Everett 1992), and exact regular equivalence (Everett and Borgatti 1994). See Section 2 for a more detailed description of the various concepts and additional references in Wasserman and Faust (1994) and Brandes and Erlebach (2005).

Since noisy empirical network data does typically not satisfy the requirements of these ideal types of partitions, the analyst also has to specify how to account for deviations from ideal structure within and between vertex classes. For instance, Batagelj, Doreian, and Ferligoj (1992) propose to compute partitions such that the least number of ties violates the condition imposed by regular equivalences. However, since the computation of the optimal partition is NP-hard in general (Fiala and Paulusma 2003), optimization algorithms as the one proposed by Batagelj et al. (1992) might get stuck in local minima and cannot be applied to large graphs.

\subsection{Contributions}

The concept of structural similarity employs a different strategy to cope with the irregularities of empirical data: rather than allowing deviations from ideal types of partitions (such as regular partitions), we no longer require that role assignments must be discrete partitions but keep the constraint for exact regular equivalences. More precisely, we relax the requirement of being a discrete partition to that of being an orthogonal projection in Euclidean space, ${ }^{1}$ while keeping the same requirement of compatibility with the network structure as for exact regular equivalences (also called $e q$ uitable partitions, see Section 2). The major advantages of structural similarities are that they ameliorate the computational intractability of discrete problem formulations, admit a precise characterization of stability with re-

1. It will be clarified in Section 3.1.1 that discrete vertex partitions can indeed be represented by specific orthogonal projections. 
spect to noise in the input data, and allow vertices to play more than one role (i.e., standing between classes) as well as being of varying importance. Furthermore, structural similarities yield efficient spectral methods thatas it turns out-generalize established spectral approaches to various graph partitioning problems.

A structural similarity yields a low-dimensional embedding for the vertices of a graph that can be further processed in two different ways. The first possibility is to apply a distance-based clustering procedure to the vertices in the low-dimensional embedding to obtain a discrete vertex partition (we illustrate this approach in Section 4). Although the final result is a discrete partition (as in blockmodeling), the intermediate step via the spectral projection yields a significant advantage over the optimizational approach to blockmodeling: it can be guaranteed that our algorithm computes an optimal result when applied to graphs drawn from specific probability distributions. Note that this has not been achieved (and seems to be hard to achieve) for local optimization algorithms, such as the one proposed by Batagelj et al. (1992).

A second way to deal with the low-dimensional embedding is not to round it to a discrete partition but rather to apply multidimensional scaling techniques to visualize the result in two or three dimensional space. Vertices that occupy similar positions (i. e., play similar roles) will then be drawn close together and vertices that occupy very different positions will be far apart. The advantages of such a continuous representation of vertex positions are its suitability for dynamically changing data (compare the specific application in Section 5) and the fact that it can accommodate vertices that stand between two or more positions (actors that play more than one role). We argue that such situations arise often in real-world data and forcing vertices to be members of one and only one class would then produce sub-optimal results.

This article is organized as follows. In Section 2, we give an overview of blockmodeling and role assignment in networks. In Section 3, we introduce our new proposal structural similarity. Section 4 presents an application of the framework of structural similarity to partitioning of random graphs that exhibit a specific hidden structure. In Section 5 we show how structural similarities are applied to the dynamic visualization of empirical networks.

Previous conference publications. Structural similarities have been first defined in Brandes and Lerner (2004). The current paper extends Brandes and Lerner (2004) in three aspects. First, the definition of structural similarities is generalized to directed graphs where the concept splits up into three different versions and where the computation is no longer done via eigen- 
vectors but via the Schur form of general real matrices. Second, the stability (robustness to noise) of structural similarities and other desirable criteria are characterized. Finally, it is shown how structural similarities relate to established spectral graph partitioning algorithms-especially it is clarified in which direction the latter are generalized. Structural similarities have been applied to the analysis and visualization of conflict networks in Brandes, Fleischer, and Lerner (2006) and Brandes and Lerner (2008) and to random graph coloring in Brandes and Lerner (2006). We review both applications in this paper since they provide a good illustration of how structural similarities can be used.

\section{Blockmodeling}

Given a graph $G=(V, E)$ with vertex set $V$ and set of (directed) edges $E \subseteq V \times V$, a role assignment of $G$ is a partition $\mathscr{P}=\left\{C_{1}, \ldots, C_{k}\right\}, C_{i} \subseteq V$ of the vertex set. Equivalently, a role assignment is a surjective mapping $r: V \rightarrow\{1, \ldots, k\}$ to a set of classes, colors, or positions. Again equivalently, a role assignment is given by an equivalence relation on the vertex set. If $\mathscr{P}=\left\{C_{1}, \ldots, C_{k}\right\}$ is a partition of $V$ and $C_{i}$ and $C_{j}$ two of its classes, then the associated block is defined by

$$
E\left(C_{i}, C_{j}\right)=E \cap C_{i} \times C_{j} .
$$

A vertex partition induces a smaller graph, called the role graph, that encodes how the vertex classes are connected. More precisely, if $G=(V, E)$ is a graph and $r: V \rightarrow W$ a role assignment, then the induced role graph or quotient $R=(W, F)$ has vertex set $W$ (the set of classes, positions, or colors) and edge set $F \subseteq W \times W$ defined by

$$
F=\{(r(u), r(v)) ;(u, v) \in E\} .
$$

See Figure 1 for a small example of a graph with a given role assignment and the induced role graph.

\subsection{Ideal Block Types}

Specific types of role assignments are defined by imposing compatibility constraints with the edges of the graph, or alternatively, requiring specific block types. Prominent specific types of vertex partitions are reviewed in the following and illustrated in Figure 2.

Two vertices are called structurally equivalent (Lorrain and White 1971) if they have identical neighborhoods, i.e., if they are connected to exactly the same other vertices. (Often loops, i. e., edges connecting vertices with themselves are disregarded, however, this distinction is of no 

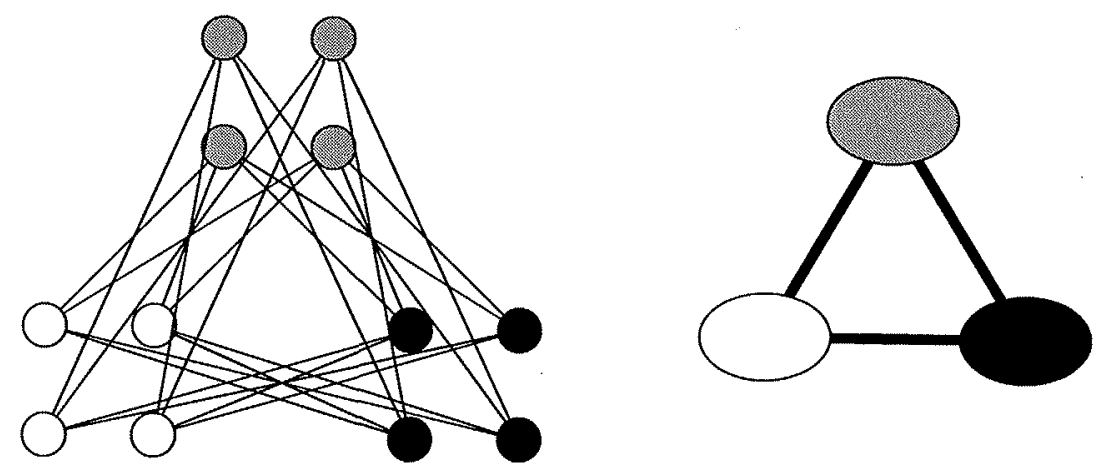

Figure 1. Left: Graph with a vertex partition, indicated by the coloring. Right: The induced role graph or quotient. In the context of structural similarities, the edges of the quotient have weight two.

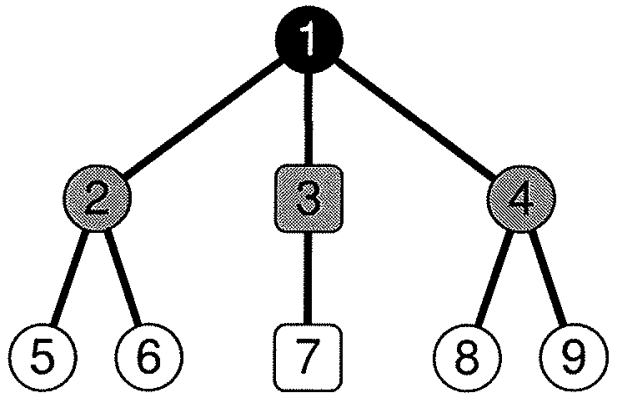

Figure 2. Example from Wasserman and Faust (1994) illustrating different types of role assignments. Only $\{5,6\}$ and $\{8,9\}$ are pairs of structurally equivalent vertices. The partition $\{1\},\{3\},\{2,4\},\{7\},\{5,6,8,9\}$ is equitable and induces an automorphic equivalence. The partition $\{1\},\{2,3,4\},\{5,6,7,8,9\}$ is regular.

importance here.) Equivalently, a partition is structural for a graph if all associated blocks $E\left(C_{i}, C_{j}\right)$ are either empty, $E\left(C_{i}, C_{j}\right)=\emptyset$, or complete, $E\left(C_{i}, C_{j}\right)=C_{i} \times C_{j}$.

Structural equivalence imposes a very strong requirement and does not well match the intuitive notion of social role or position. This has been pointed out by Sailer (1978), who remarked that two individuals should be considered to occupy the same social position, if they are related to the same other positions but not necessarily to the same individuals. For instance, two judges might be considered as similar due to having similar relations to other judges, lawyers, etc., without knowing any individual in common. Other papers illustrating the insufficiency of structural equivalence include Borgatti and Everett (1992) and Luczkovich, Borgatti, Johnson, and Everett (2003). To overcome these drawbacks of structural equivalence, several other classes 
of partitions have been proposed that require equivalent (but not necessarily identical) neighborhoods for equivalent vertices. A very general result clarifying under what conditions are partitions compatible with the concatenation of tie relations is given by Kim and Roush (1984); an overview of various types of partitions that generalize structural equivalences is given in Pattison (1993). In the following we review generalizations of structural equivalence that are most relevant for this paper.

Two vertices $u$ and $v$ are called automorphically equivalent (Everett and Borgatti 1994) if there is a graph automorphism mapping $u$ to $v$. (An automorphism $\varphi$ of a graph $G=(V, E)$ is a permutation of $V$ such that every two vertices $u, v$ are adjacent if and only if their images $\varphi(u), \varphi(v)$ are adjacent.) Two automorphically equivalent vertices cannot be distinguished by any structural property of the graph (Borgatti and Everett 1992). Irregular empirical networks are unlikely to admit interesting, non-trivial automorphisms and their computation is inefficient anyway.

A partition is called exact regular (Everett and Borgatti 1994) if for every two of its classes $C_{1}$ and $C_{2}$, all vertices in $C_{1}$ have the same number of neighbors in $C_{2}$. Equivalently, a partition is exact regular if for every associated block $B$, there are two numbers $r_{B}$ and $c_{B}$ such that all rows of $B$ sum up to $r_{B}$ and all columns of $B$ sum up to $c_{B}$. Exact regular partitions are also known as divisors of graphs (Cvetković, Doob, and Sachs 1995) and equitable partitions (Godsil and Royle 2001).

A partition is called regular (White and Reitz 1983; Everett and Borgatti 1994) if for every two of its classes $C_{1}$ and $C_{2}$ it holds that, whenever one vertex in $C_{1}$ has a neighbor in $C_{2}$, then every vertex in $C_{1}$ has a neighbor in $C_{2}$. Equivalently, a partition is regular if every block is either empty or it has at least one non-zero entry in every row and in every column. Regular equivalences are also called stable partitions (Paige and Tarjan 1987) and are closely related to bisimulations in the theory of finite state automaton (Milner 1980).

The abovementioned types of role assignments are ordered in decreasing strength in the sense that a structural partition is automorphic, an automorphic partition is equitable, and an equitable partition is regular; the inclusions are all proper. A big conceptual difference is between structural equivalence and the other three: structural equivalence requires identical neighborhoods for equivalent vertices, whereas the other formulations require only equivalent (e. g., same-colored) neighborhoods; compare Borgatti and Everett (1992) and see Figure 3 for illustration.

Generalized blockmodeling (Batagelj 1997; Doreian et al. 2005) is not restricted to the abovementioned types of role assignments (structural, automorphic, exact regular, and regular) but specifies more block types (e. g., 

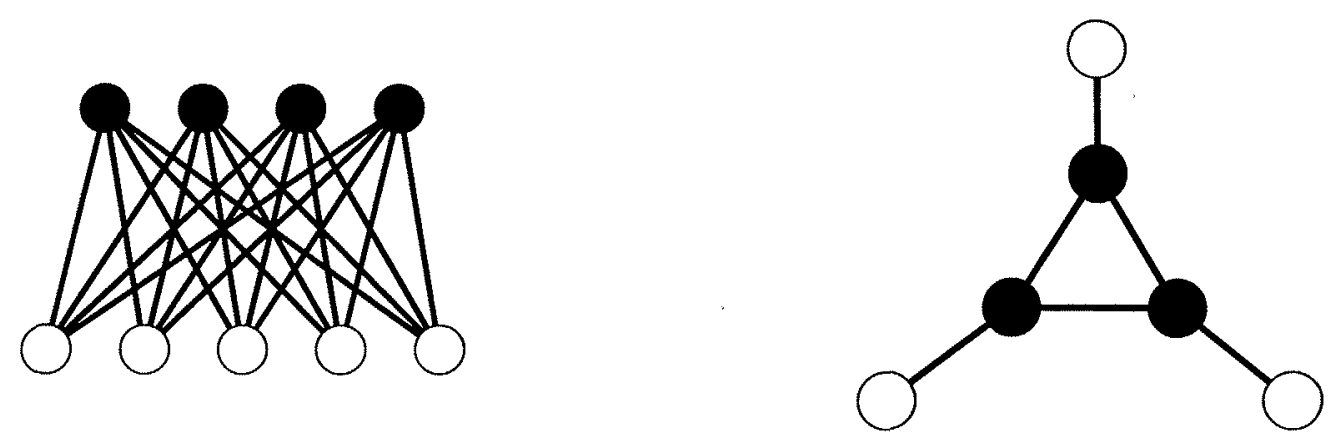

Figure 3. Two graphs with vertex partitions indicated by the coloring. Left: Equivalent vertices have identical neighborhoods. Right: Equivalent vertices have equivalent but nonidentical neighborhoods.

row-regular, column-regular, row-dominant, column-dominant, to name a few). Furthermore, in generalized blockmodeling the block types associated to a specific partition need not be all of the same type; for instance, one could allow blocks to be either regular, row-dominant, or zero and prohibit all other block types. Prespecified blockmodeling (Doreian et al. 2005) is the task of computing a vertex partition so that the resulting blockmodel is of a prespecified structure, i. e., that the blocks between specific classes belong to a fixed type.

\subsection{Relaxation of Ideal Block Types}

The constraints made for regular, exact regular, automorphic, or structural equivalence define ideal (unperturbed) types of role assignment. These constraints are typically not strictly satisfied in irregular application data so that the ideal types need to be relaxed appropriately. Such a relaxation can be made in two different directions: allowing that a few ties violate the constraints, as this is done in optimizational approaches to blockmodeling (Batagelj et al. 1992), or relaxing the requirement that role assignments have to be discrete partitions, as will be done in this article.

\subsubsection{An Optimizational Approach}

In a nutshell, Batagelj et al. (1992); Doreian et al. (2005) specify error functions that measure the deviation between ideal block types and observed blocks. For instance, the deviation between an observed block $B$ and a complete block is the number of zeros in $B$, the deviation between $B$ and a zero block is the number of ones in $B$, the deviation between $B$ 
and a non-zero regular block is the number of zero rows plus the number of zero columns, etc. Given a number $k$ of classes, it is then attempted to compute a $k$-partition that minimizes the sum of errors over all $k^{2}$ blocks. A drawback of this approach is that the minimization is NP-hard in general (Fiala and Paulusma 2003) so that one has to rely on local search algorithms that cannot guarantee to actually find a global optimum.

\subsubsection{Our Approach}

As it has been outlined in the introduction, structural similarities relax the condition that role assignments must be discrete vertex partitions but keep the requirement for exact regular equivalences. The definition, characterization, computation, as well as various desirable properties of structural similarities are detailed in Section 3. Concrete application areas for the framework of structural similarities are presented in Section 4 and Section 5.

\section{Structural Similarity}

\subsection{Definition of Structural Similarity}

\subsubsection{Relaxing Equivalence}

We represent a partition $\mathscr{P}$ of $n$ vertices into $k$ classes by its characteristic matrix $P$, which is defined to be the $k \times n$ matrix

$$
P_{c v}= \begin{cases}1 / \sqrt{r} & \text { if } v \text { is in class } c \text { of size } r \\ 0 & \text { if } v \text { is not in class } c\end{cases}
$$

The normalization by $\sqrt{r}$ seems to be arbitrary at the moment. However, it ensures that the rows have unit length in the Euclidean norm, which simplifies formulas later in this paper.

The characteristic matrices of partitions satisfy $P P^{\mathrm{T}}=\mathrm{id}_{k}$ and have the property that in each column there is exactly one entry different from zero, since each vertex is in exactly one class. To define relaxations of vertex partitions we drop the restriction on the columns (i.e., we allow vertices to be members of several classes), but keep the normalization requirement $P P^{\mathrm{T}}=\mathrm{id}_{k}$, which ensures that rows are orthogonal and have unit length. The real value in row $c$ and column $v$ is called the degree of membership of vertex $v$ to class $c$.

Definition 1. A real $k \times n$ matrix $P$ is called a projection (of rank $k$ ), if $P P^{\mathrm{T}}=\mathrm{id}_{k}$. 
The definition of the characteristic matrix of a partition yields a natural embedding of the set of partitions into the set of projections. The rank of a projection corresponds to the number of classes of a partition. The position of a vertex $v$ is defined to be the $v^{\prime}$ th column of $P$, denoted by $P(v)$, i. e., a linear combination of classes. The importance of a vertex $v$ is defined to be $\|P(v)\|=\sqrt{P_{1 v}^{2}+\cdots+P_{k v}^{2}}$. It is a measure for the prominence or involvement of $v$ in the role assignment defined by $P$.

A similar relaxation process is performed for equivalence relations. We represent an equivalence relation $\sim$ on a set of $n$ vertices by its characteristic matrix $S$, which is defined to be the $n \times n$ matrix

$$
S_{u v}= \begin{cases}1 / r & \text { if } u \sim v \text { and } r \text { is the size of } v \text { 's equivalence class; } \\ 0 & \text { if } u \nsim v .\end{cases}
$$

The specific normalization by $r$ has been chosen such that similarities are in a one-to-one correspondence with projections-see below.

The characteristic matrices of equivalence relations satisfy $S^{\mathrm{T}}=S$ and $S^{2}=S$ (due to the symmetry and transitivity of equivalence relations) and have the property that - after appropriate reordering of rows and columnsthey have block-diagonal form with constant diagonal blocks (since vertices are "equally equivalent" to all members of their class and not equivalent to vertices outside their class). To define relaxations of equivalence relations we drop the restriction on the block-diagonal form but keep the requirements $S^{\mathrm{T}}=S$ and $S^{2}=S$. The real value $S_{u v}$ in row $u$ and column $v$ is called the similarity of vertices $u$ and $v$.

Definition 2. An $n \times n$ matrix $S$ is called a similarity if it is symmetric, i. e. $S^{\mathrm{T}}=S$, and idempotent, i. e. $S^{2}=S$. The rank of $S$ is defined to be the number of non-zero eigenvalues of $S$ and, hence, is identical with its rank as a matrix.

For a fixed integer $n$-the number of vertices of the graph - similarities of rank $k$ correspond to orthogonal projections to $k$-dimensional subspaces of $\mathbb{R}^{n}$, compare Artin (1991).

The definition of the characteristic matrix of an equivalence relation yields a natural embedding of the set of equivalence relations into the set of similarities. The rank of a similarity corresponds to the number of equivalence classes of the equivalence relation.

Like equivalence relations and their associated partitions, similarities and projections are just two points of view of the same concept, in the sense that every projection induces a similarity and vice versa. A projection $P$ induces a similarity $S_{P}$ by setting $S_{P}=P^{\mathrm{T}} P$. This implies that, for a pair of vertices $u$ and $v$, the similarity between $u$ and $v$ is defined to be $\langle P(u), P(v)\rangle$, 
i. e., the inner product of the two position vectors. Thus, similarity is high if $u$ and $v$ belong to a large degree to the same classes and it is low if $u$ and $v$ belong to (almost) disjoint sets of classes. Conversely, let $S$ be a similarity of rank $k$. Then, its image img $S$ (i. e., the space spanned by its column vectors) has dimension $k$. Let $v_{1}, \ldots, v_{k} \in \mathbb{R}^{n}$ be any orthonormal basis of img $S$. Then the $k \times n$ matrix $P_{S}$ whose rows are the $v_{i}, i=1, \ldots, k$ is a projection with the property $P_{S}^{\mathrm{T}} P_{S}=S$.

Projections are only unique up to orthogonal basis transformations of their $k$-dimensional image space; see Artin (1991) for a definition of orthogonal matrices and their relationship to basis transformations. For instance, if a projection $P$ is the characteristic matrix of a discrete partition $\mathscr{P}$ then a permutation of the classes of $\mathscr{P}$ yields a different projection $P^{\prime}$ that can be obtained from $P$ by a permutation of the $k$ rows. For the general case, let $P$ and $P^{\prime}$ be two projections of dimension $k \times n$; it is straightforward to show that the following two assertions are equivalent.

1. $P^{\mathrm{T}} P=P^{\prime \mathrm{T}} P^{\prime}$, i. e., $P$ and $P^{\prime}$ define the same similarity on $n$ vertices.

2. There is an orthogonal $k \times k$ matrix $\varphi$ such that $\varphi P^{\prime}=P$.

In either case we do not distinguish between $P$ and $P^{\prime}$. With this convention, the two mappings $S \mapsto P_{S}$ and $P \mapsto S_{P}$ are mutually inverse (Brandes and Lerner 2004). That is, for each similarity there is a unique associated projection (up to the choice of a basis) and vice versa.

Since projections are only unique up to orthogonal rotations of their image space, one can rotate the rows of the projection matrix such that some criterion function is optimized. An established criterion is the so-called varimax objective function (Kaiser 1958); also compare Van de Velden and Kiers (2005). This criterion yields a row-rotated projection matrix such that its entries are (optimally) either large or close to zero. Such a matrix can be seen as being close to a discrete partition (vertices are in a specific class if their degree of membership is large) and is thus appropriate if discrete partitions are the goal of the analysis.

\subsubsection{Quotients of Graphs Under Projections}

A graph together with a vertex partition induces a smaller graph on the set of classes, called the role graph or quotient (compare Section 2). Likewise, projections (and thus similarities) reduce graphs to smaller factors called quotients that are graphs with weighted edges on the set of classes.

Definition 3. Let $G$ be a graph (potentially with edge weights) with adjacency matrix $A \in \mathbb{R}^{n \times n}$ and $P \in \mathbb{R}^{k \times n}$ a projection. Then, $G$ and $P$ induce a $k \times k$ matrix $B$ by setting $B=P A P^{\mathrm{T}}$. The graph $G / P$ on $k$ vertices that is determined by its adjacency matrix $B$ is called the quotient of $G$ modulo $P$. 
If a projection is induced by a discrete partition then the weight of the edge between two classes $c_{1}$ and $c_{2}$ is, thus, equal to the number of edges connecting $c_{1}$ and $c_{2}$ divided by $\sqrt{\left|c_{1}\right| \cdot\left|c_{2}\right|}$. As an example, see Figure 1 , where the white and black class in the quotient are connected by an edge of weight two, since white vertices have on average two black neighbors.

\subsubsection{Compatibility with the Graph Structure}

All that remains to do to define structural similarities is to translate the constraint that "equivalent vertices must have equivalent neighborhoods" to the larger family of similarities-where we can no longer speak of "equivalent" or "non-equivalent." This is achieved by generalizing matrix equations that are fulfilled by exact regular equivalence relations (equitable partitions) to the larger family of similarities. We show in Theorem 2 that we indeed generalize equitable partitions.

For directed graphs we distinguish between similarities that are structural with respect to outgoing edges, incoming edges, and both. It is easy to see that for undirected graphs (symmetric adjacency matrix $A$ ) all three definitions coincide, and yield the definition of structural similarities given in Brandes and Lerner (2004).

Definition 4. Let $G$ be a graph with adjacency matrix $A \in \mathbb{R}^{n \times n}, P \in \mathbb{R}^{k \times n}$ a projection, and $B=P A P^{\mathrm{T}}$ the adjacency matrix of the induced quotient. Then, $P$ and its associated similarity is called

1. out-structural if $P A=B P$,

2. in-structural if $P A^{\mathrm{T}}=B^{\mathrm{T}} P$,

3. structural if both conditions hold.

For example, in Figure 1 every white vertex has exactly two black neighbors. Thus, the number of black neighbors of each white vertex is equal to the average number of black neighbors of white vertices, i. e., equal to the weight of the edge between the white and black class in the quotient. It is this equality which is required in the matrix equations in Definition 4.

\subsection{Characterizing Structural Similarities}

Just looking at Definition 4, it seems to be hard to actually determine structural similarities for a given graph. Next, we derive equivalent conditions for a similarity to be structural. These conditions yield efficient, numerical algorithms to compute structural similarities and serve to relate structural similarities to traditional spectral graph partitioning methods.

A subspace $U \subseteq \mathbb{R}^{n}$ is called $A$-invariant if $A u \in U$ for all $u \in U$. If $A$ is a symmetric matrix (for instance, the adjacency matrix of an undirected 
graph), then the $A$-invariant subspaces coincide with the subspaces spanned by eigenvectors of $A$ (Artin 1991). The kernel of a matrix $A$ is the set of vectors that are mapped to zero, i. e., $\operatorname{ker} A=\{v ; A v=0\}$.

Theorem 1. Let $G$ be a graph with adjacency matrix $A \in \mathbb{R}^{n \times n}$ and $S \in \mathbb{R}^{n \times n}$ a similarity. Then

1. $S$ is out-structural iff $\operatorname{ker} S$ is A-invariant iff $S A=S A S$ holds,

2. $S$ is in-structural iff img $S$ is A-invariant iff $A S=S A S$ holds,

3. $S$ is structural iff img $S$ and $\operatorname{ker} S$ are A-invariant iff $A S=S A$ holds.

Proof: Let $P=P_{S}$ be the projection associated to $S$ and $B=P A P^{\mathrm{T}}$. For proving the first assertion, assume $S A=S A S$ and multiply this equation with $P$ from the left; this gives us $P P^{\mathrm{T}} P A=P P^{\mathrm{T}} P A P^{\mathrm{T}} P$, implying $P A=B P$, i. e., $P$ is out-structural. Conversely, given $P$ with $P A=B P$ we get

$$
S A=P^{\mathrm{T}} P A=P^{\mathrm{T}} B P=P^{\mathrm{T}} P A P^{\mathrm{T}} P=S A S .
$$

Now assume that $\operatorname{ker} S$ is not $A$-invariant. Then there is a $v \in \mathbb{R}^{n}$ such that $S(v)=0$ but $S A(v) \neq 0$. It follows that $S A(v) \neq 0=S A S(v)$ and $S A \neq S A S$. Conversely, assume that $\operatorname{ker} S$ is $A$-invariant and let $v \in \mathbb{R}^{n}$. The space $\mathbb{R}^{n}$ is the orthogonal sum of $\operatorname{img} S$ and $\operatorname{ker} S$ (since $S$ is symmetric). This implies that $v=u_{1}+u_{2}$ for some $u_{1} \in \operatorname{ker} S$ and $u_{2} \in \operatorname{img} S$. Then, since by assumption $A\left(u_{1}\right) \in \operatorname{ker} S$, we get $S A(v)=S A\left(u_{2}\right)=S A S(v)$ and it follows that $S A=S A S$.

The second assertion follows from the first by considering $A^{\mathrm{T}}$ and $B^{\mathrm{T}}$ and using the fact that img $S$ is $A$-invariant if and only if $\operatorname{ker} S$ is $A^{\mathrm{T}}$-invariant (Artin 1991). The third assertion is obtained by the former two.

We show next that, if a projection $P$ is induced by a discrete partition $\mathscr{P}$, then $P$ is structural if and only if $\mathscr{P}$ is equitable. Thus structural similarities are indeed relaxations of equitable partitions. Taking the notation from Godsil (1993), a vertex coloring of a directed graph is called row equitable if for every two color classes, say red and blue, all red vertices have the same number of outgoing edges to blue vertices; for the definition of column equitable change "outgoing" to "incoming;" a coloring is equitable if it is both row equitable and column equitable.

Theorem 2. Let $G=(V, E)$ be a graph with adjacency matrix $A$ and $P$ the characteristic matrix of a partition $\mathscr{P}$ of $V$. Then $P$ is an out-structural projection if and only if $\mathscr{P}$ is row equitable, $P$ is in-structural if and only if $\mathscr{P}$ is column equitable, and $P$ is structural if and only if $\mathscr{P}$ is equitable.

Proof (sketch): Assume that $\mathscr{P}$ is an equitable partition, $\sim$ its associated equivalence relation, and $S$ the characteristic matrix associated to $\sim$. Let 
$u, v \in V, r_{u}=|[u]|$ (i. e., $r_{u}$ is the size of $u$ 's equivalence class) and $r_{v}=|[v]|$. Further, let

$$
R_{u v}=\sum_{\substack{w^{\prime}: w^{\prime} \sim v \\ w: w \sim u}} A_{w w^{\prime}}
$$

denote the number of edges connecting the class of $v$ with the class of $u$. A central observation is that, since $\sim$ is out-equitable, every $w^{\prime} \sim v$ gets the same share of $R_{u v}$; in particular,

$$
\sum_{w: w \sim u} A_{w v}=R_{u v} / r_{v}
$$

Likewise, since $\sim$ is in-equitable, every $w \sim u$ gets the same share of $R_{u v}$; in particular,

$$
\sum_{w^{\prime}: w^{\prime} \sim v} A_{u w^{\prime}}=R_{u v} / r_{u}
$$

Together we obtain

$$
(S A)_{u v}=\sum_{w \in V} S_{u w} A_{w v}=\sum_{w: w \sim u} \frac{1}{r_{u}} A_{w v}=\frac{1}{r_{u} r_{v}} R_{u v},
$$

and

$$
(A S)_{u v}=\sum_{w^{\prime} \in V} A_{u w^{\prime}} S_{w^{\prime} v}=\sum_{w^{\prime}: w^{\prime} \sim v} \frac{1}{r_{v}} A_{u w^{\prime}}=\frac{1}{r_{u} r_{v}} R_{u v},
$$

from which it follows $S A=A S$, i. e., $S$ is structural.

The converse implication can be obtained by reverting this argument and the assertions for out-structural and in-structural follow by similar techniques (details are omitted).

\subsubsection{Computation of Structural Similarities}

As corollaries of Theorem 1 we can give the following general algorithms for the computation of structural similarities. We distinguish between undirected and directed graphs. The algorithms below are generic in the sense that they involve the selection of a subset of eigenvalues that is not restricted here. Which subset actually has to be chosen depends on what the similarity is for, what properties it should satisfy, or what criterion should be optimized. See Sections 3.3 and 3.4 for such restricting properties and Sections 4 and 5 for application examples.

Undirected Graphs. From Theorem 1 we get that for undirected graphs, a similarity $S$ is structural if and only if its image is generated by eigenvectors of $A$. Thus, a general way for determining any structural similarity is the following. 
1. Compute the eigenvectors of the graph's adjacency matrix.

2. Select a subset of $k$ (orthogonal and normalized) eigenvectors and write them into the rows of a matrix $P$.

$P$ is a structural projection and $S=P^{\mathrm{T}} P$ a structural similarity.

For efficient computation of eigenvectors see, e.g., Golub and van Loan (1996). As an example, the (equitable) partition in Figure 1 is associated to the structural projection onto eigenvectors with eigenvalues $4,-2$, and -2 .

Directed Graphs. A representation of all invariant subspaces of a graphand thus of all (out-/in-)structural similarities-is obtained by the Jordan form of the graph's adjacency matrix. See Gohberg, Lancaster, and Rodman (1986) for a comprehensive treatment of this topic and Golub and van Loan (1996) for algorithms. However, the Jordan form is highly sensitive to perturbations and thus not suitable for the applications of structural similarity that we envision here. In Section 3.4 we identify a subset of structural similarities (called simple) that are robust to noise and have other desirable properties. All simple in-structural (out-structural) similarities can be computed by the following procedure. (For the definition of the real Schur form see, e. g., Golub and van Loan (1996).)

1. Compute a real Schur form $X=Q^{\mathrm{T}} A Q$ of the graph's adjacency matrix A.

2. Select a subset of $k$ diagonal elements of $X$ and reorder the Schur form such that these values are at the upper left (lower right) position. This yields the Schur form $X^{\prime}=Q^{\prime \mathrm{T}} A Q^{\prime}$.

3. Write the $k$ leftmost (rightmost) columns of $Q^{\prime}$ in the rows of a matrix $P$.

$P$ is an in-structural (out-structural) projection and $S=P^{\mathrm{T}} P$ an in-structural (out-structural) similarity.

Efficient computation and reordering of the Schur form is treated in Golub and van Loan (1996). Eigenvalues of unsymmetric real matrices can be complex. In this case, complex conjugate pairs of eigenvalues must be kept together in Step 2; see Golub and van Loan (1996) for details.

\subsubsection{Structural Similarities Compared to Established Spectral Methods}

Orthogonal projections to low-dimensional subspaces that are spanned by eigenvectors are a frequent tool in many data analysis and graph partitioning applications. Concrete examples include latent semantic indexing $(\mathrm{Pa}-$ 
padimitriou, Raghavan, Tamaki, and Vempala 2000), Web search (Achlioptas, Fiat, Karlin, and McSherry 2001), collaborative filtering (Azar, Fiat, Karlin, McSherry, and Saia 2001), learning mixtures of distributions (Vempala and Wang 2002), analysis of the autonomous systems graph (Gkantsidis, Mihail, and Zegura 2003), graph clustering (Kannan, Vempala, and Vetta 2004), random graph coloring (Alon and Kahale 1997), spectral graph partitioning (McSherry 2001), and spectral graph bisecting (Coja-Oghlan 2005).

Typically, these methods project onto the eigenvectors associated to the (few) eigenvalues with the largest absolute values. (We will refer to these methods as traditional spectral methods in the following.) Thus, by Theorem 1, these methods compute special cases of structural similarities; the latter are not restricted to projecting to the largest eigenvalues but can choose all subsets.

We argue below that the difference between these two approaches is conceptually the same as between the requirements of identical vs. equivalent neighborhoods for equivalent vertices (compare Section 2). Thus, traditional spectral methods can be seen as relaxations of structural equivalence (Lorrain and White 1971), whereas structural similarities have been characterized as relaxations of the-much more general-class of exact regular equivalence (see Theorem 2).

An Illustrating Example. For instance, the structural partition shown in Figure 3 (left) can be computed by projecting to the two eigenvalues \pm 4.47 , which have the maximal absolute values (the others are a seven-fold eigenvalue at 0 ). In contrast, the equitable partition shown in Figure 3 (right) can be computed by projecting to the two eigenvalues 2.41 and -0.41 , out of the set of eigenvalues

$$
\mathbf{2 . 4 1}, 0.62,0.62,-0.41,-1.62,-1.62 \text {. }
$$

Thus, restricting spectral algorithms to projections to the maximal eigenvalues yields methods that cannot even determine some, intuitively outstanding, automorphic equivalences.

The General Case. Let $A$ be the adjacency matrix of an undirected graph, $S$ the matrix of a structural similarity for this graph, and $u$ and $v$ two vertices. The value $\|A(u-v)\|=\|A(u)-A(v)\|$ is a measure for the difference of the neighborhoods of $u$ and $v$. Thus, $u$ and $v$ have almost identical neighborhoods if $\|A(u-v)\|$ is small. Similarly, $u$ and $v$ are considered as almost equivalent by $S$ if $\|S(u-v)\|$ is small. We clarify below that traditional spectral methods optimize the property $\|S(u-v)\|$ is small if and only if $\|A(u-v)\|$ is small, i. e., 
$u$ and $v$ are considered as almost equivalent by $S$ if and only if $u$ and $v$ have almost identical neighborhoods.

To make this precise, let $x_{1}, \ldots, x_{n}$ be orthonormalized eigenvectors of $A$ with associated eigenvalues $\lambda_{1}, \ldots, \lambda_{n}$ which are ordered such that $S$ projects to the first $k$ eigenvectors. (Thus, if $S$ is determined by traditional spectral methods the first $k$ eigenvalues are those with maximal absolute values.) Further, let $c_{1}$ and $c_{2}$ be defined by

$$
c_{1}=\max _{i=1, \ldots, k} 1 /\left|\lambda_{i}\right|, \quad c_{2}=\max _{i=k+1, \ldots, n}\left|\lambda_{i}\right| .
$$

(Note that $c_{1}$ is defined only if $S$ does not project to an eigenvalue $\lambda_{i}=0$, which can be safely assumed for traditional spectral methods.) If $k$ is given, then traditional spectral methods chose the structural projection of dimension $k$ that minimizes $c_{1}$ and $c_{2}$ over all structural projections of dimension $k$. Let $y$ be any vector of norm less than or equal to $\sqrt{2}$ and $y=\sum_{i=1}^{n} a_{i} x_{i}$ for uniquely determined real values $a_{i}$. It is

$$
\begin{aligned}
& \|S(y)\|^{2}=\cdot \sum_{i=1}^{k} a_{i}^{2} \leq c_{1}^{2} \sum_{i=1}^{k}\left(a_{i} \lambda_{i}\right)^{2} \leq c_{1}^{2}\|A(y)\|^{2} \quad \text { and } \\
& \|A(y)\|^{2}=\sum_{i=1}^{k}\left(a_{i} \lambda_{i}\right)^{2}+\sum_{i=k+1}^{n}\left(a_{i} \lambda_{i}\right)^{2} \leq\|A\|_{2}^{2}\|S(y)\|^{2}+2 c_{2}^{2} .
\end{aligned}
$$

By taking $y=u-v$ for the two vertices $u$ and $v$, we obtain from (1) and (2) the following two properties for a structural similarity $S$.

1. Assume that $S$ does not project to an eigenvalue $\lambda_{i}=0$. If $\| A(u)-$ $A(v) \|$ is small, then $\|S(u)-S(v)\|$ is small, i. e., vertices with almost identical neighborhoods are considered as almost equivalent by $S$. Furthermore, the ratio $\|S(u-v)\| /\|A(u-v)\|$ is bounded from above by $c_{1}$ which is minimized by traditional spectral methods.

2. Conversely, if $\|S(u)-S(v)\|$ is small then $\|A(u)-A(v)\|$ is bounded by $\sqrt{2} c_{2}$ plus a small $\varepsilon>0$, i. e., if vertices are seen as almost equivalent by $S$, then their neighborhoods can differ by no more than $\sqrt{2} c_{2}$. Traditional spectral methods minimize $c_{2}$, i. e., those methods recognize only vertices with almost identical neighborhoods as almost equivalent.

It is important to note that these two properties can cause traditional spectral methods to miss some structure of the graph. Vertices may have a high structural similarity (e. g., they may be even automorphically equivalent) without having almost identical neighborhoods; compare Figure 3. 


\subsection{Selecting Structural Similarities}

Until now the definition of structural similarities yields a huge set of possible choices for each given graph. Theorem 3 characterizes, for the case of undirected graphs, all structural similarities that induce a prespecified quotient-similar as in prespecified blockmodeling Doreian et al. (2005). (See Artin (1991) for a definition of the characteristic polynomial of a square matrix. Note that the characteristic polynomial of a matrix $B$ divides the characteristic polynomial of a matrix $A$ if and only if the eigenvalues of $B$ are a subset, counting multiplicities, of the eigenvalues of $A$.)

Theorem 3. (Brandes and Lerner (2004)) Let $G$ be a graph with symmetric adjacency matrix $A \in \mathbb{R}^{n \times n}$ and $R$ be a graph with adjacency matrix $B \in$ $\mathbb{R}^{k \times k}$. Then there is a structural projection $P \in \mathbb{R}^{k \times n}$ such that $B=P A P^{\mathrm{T}}$ if and only if $B$ is symmetric and the characteristic polynomial of $B$ divides the characteristic polynomial of $A$. In this case, the image of the associated similarity $S_{P}$ is generated by eigenvectors of $A$ associated to the eigenvalues of $B$.

For example, the partition in Figure 1 corresponds to the structural projection onto eigenvalues $4,-2$, and -2 . These are exactly the eigenvalues of the quotient. An application illustrating the usage of Theorem 3 is presented in Section 5 .

\subsection{Properties of Simple Structural Similarities}

We define here a subset of structural similarities (called simple) and show that these enjoy two desirable properties, that of being invariant under automorphisms and that of being robust against perturbations of the adjacency matrix. These criteria clarify which structural similarities are applicable to the analysis of noisy, empirical data.

We need some basic facts concerning invariant subspaces. Let $A$ be the adjacency matrix of a graph on $n$ vertices and $S$ a similarity on that graph. If $U=\operatorname{ker} S(U=\operatorname{img} S)$ is a $k$-dimensional $A$-invariant subspace, i.e., if $S$ is out-structural (in-structural), then the representation of $A$ with respect to the decomposition $\mathbb{R}^{n}=U \oplus U^{\perp}$ is

$$
\left[\begin{array}{cc}
B_{1} & C \\
0 & B_{2}
\end{array}\right]
$$

for matrices $B_{1} \in \mathbb{R}^{k \times k} B_{2} \in \mathbb{R}^{(n-k) \times(n-k)}$, and $C \in \mathbb{R}^{k \times(n-k)}$. An invariant subspace $U$ is called simple (Stewart and Sun 1990) if, in the notation of (3), the spectra of $B_{1}$ and $B_{2}$ have empty intersection. 

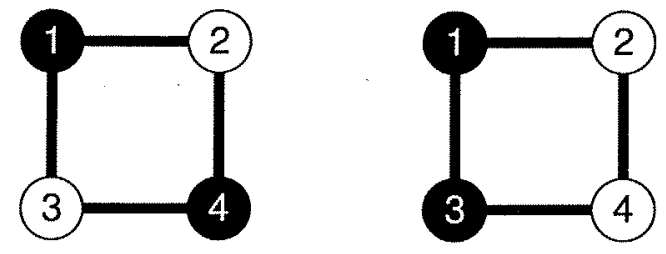

Figure 4. Two different equitable partitions on a graph with spectrum $\{2,0,0,-2\}$. Left: The coloring corresponds to the structural projection onto $\{2,-2\}$ and is automorphism invariant. This coloring reflects the unique bipartition of the graph and is therefore well justified by the graph structure. Right: The coloring corresponds to a structural projection onto $\{2,0\}$ (only one eigenvector with eigenvalue 0 is taken). This coloring is not automorphism invariant (e. g., transposing 2 and 3 changes the partition). Intuitively, it seems to be arbitrary and not justifiable by the graph structure that Vertex 1 should be more similar to 3 than to 2 , as suggested by the partition on the right.

Definition 5. A similarity $S$ is called simple, if it is

1. out-structural and $\operatorname{ker} S$ is a simple invariant subspace,

2. in-structural and $\operatorname{img} S$ is a simple invariant subspace,

3. structural and $\operatorname{ker} S$ or $\operatorname{img} S$ (and hence both) are simple invariant subspaces.

\subsubsection{Non-arbitrariness}

We say that a similarity (and hence more specifically a partition) is non-arbitrary if it is only derived from the graph's structure and not from a particular labeling of vertices. This, in turn, is formalized by being invariant under graph automorphisms, where invariant under an automorphism $\varphi$ means that the similarity of every pair of vertices $u$ and $v$ is the same as the similarity of their images $\varphi(u)$ and $\varphi(v)$ (see Definition 6). Figure 4 shows a small network together with an automorphism invariant partition $(l e f t)$ and a partition that is not automorphism invariant (right).

Definition 6. Let $G=(V, E)$ be a graph. A similarity $S$ is called automorphism invariant (for $G$ ) if for every two vertices $u, v \in V$ and every graph automorphism $\varphi: V \rightarrow V$ of $G, S_{u v}=S_{\varphi(u) \varphi(v)}$.

Theorem 4. A simple structural similarity is automorphism invariant.

Proof: Let $A$ be the adjacency matrix of a graph $G, S$ a simple structural similarity for $G$, and $X$ the permutation matrix of an automorphism of $G$. Since $S$ is a structural similarity, it follows that $\operatorname{ker} S$ and $\operatorname{img} S$ are both generated by eigenvectors of $A$. Since $S$ is simple, the eigenvalues associated to 
eigenvectors in $\operatorname{ker} S$ are pairwise disjoint to those associated to eigenvectors in img $S$. Since $X$ is an automorphism, it commutes with $A$. Hence, by Gohberg et al. (1986, Theorem 9.1.1), the image of an eigenvector $v$ of $A$ under $X$ is contained in the span of all eigenvectors associated to the same eigenvalue as $v$. Together it follows that $X$ leaves both $\operatorname{ker} S$ and $\operatorname{img} S$ invariant. Since $S$ is constant on both $\operatorname{ker} S$ (it is the zero mapping on $\operatorname{ker} S$ ) and img $S$ (it is the identity on img $S$ ), it follows that $X^{\mathrm{T}} S X=S$ and therefore that $S$ is invariant under $X$.

\subsubsection{Stability}

A second characterization is that simple similarities are exactly those that satisfy a local stability criterion. The next definition is best understood if one keeps in mind the usual definition of continuity of functions (i. e., a function $f$ is continuous in $x_{0}$ if for every $\varepsilon>0$ there is a $\delta>0$ such that $\left|x-x_{0}\right|<\delta$ implies $\left.\left|f(x)-f\left(x_{0}\right)\right|<\varepsilon\right)$.

Definition 7. Let $A$ be the adjacency matrix of a graph. A similarity $S$ that is out-structural (in-structural) for $A$ is called stable, if for every sufficiently small $\varepsilon>0$, there exists a $\delta>0$ such that for any matrix $A^{\prime}$ with $\left\|A-A^{\prime}\right\|<$ $\delta$, there is a unique similarity $S^{\prime}$ that is out-structural (in-structural) for $A^{\prime}$ and for which it holds that $\left\|S-S^{\prime}\right\|<\varepsilon$.

Loosely spoken, a stable similarity $S$ changes only slightly, if the adjacency matrix changes only slightly. Conversely, if a similarity $S$ is not stable then there are arbitrarily small perturbations of the adjacency matrix such that $S$ necessarily changes by more than a fixed positive bound.

Theorem 5. An out-structural (in-structural) similarity is stable if and only if it is simple.

Proof: For the definitions of Lipschitz stable invariant subspace and root space see Gohberg et al. (1986). Let $S$ be out-structural. Then $S$ is stable if and only if ker $S$ is a Lipschitz stable invariant subspace. By Gohberg et al. (1986, Theorem 15.5.1) this holds if and only if $\operatorname{ker} S$ is the sum of root spaces, which is true if and only if $S$ is simple.

Stable similarities, as defined in Definition 7, depend continuously on the input data. This is a minimal requirement for any measure that is applicable to noisy data. However, the ratio of change in the similarity over change in the data can still be very large. Here we define a stability measure that bounds this ratio. The following definition is an adaptation of the separator, known in matrix perturbation theory, see Stewart and Sun (1990). 
Definition 8. Let $S$ be a simple similarity and let (3) denote the corresponding decomposition of $A$. The stability of $S$ is defined to be the positive real number

$$
\sigma(S)=\min \frac{\left\|B_{1} X-X B_{2}\right\|}{\|X\|}
$$

where the minimum is taken over all matrices $X \in \mathbb{R}^{k \times(n-k)}$ that are not identical to zero. For symmetric $A$ the stability can equivalently be defined by

$$
\sigma(S)=\min \left|\lambda_{1}-\lambda_{2}\right|
$$

where the minimum is taken over all eigenvalues $\lambda_{1}$ of $B_{1}$ and $\lambda_{2}$ of $B_{2}$, see Stewart and Sun (1990).

The stability $\sigma(S)$ can be computed efficiently. For undirected graphs, this follows directly from Definition 8, for the general case see, e. g., Bai, Demmel, and McKenney (1993).

A large value $\sigma(S)$ guarantees resistance to perturbations. Many error bounds can be given that differ in the matrix norms that are used to measure the deviation, the symmetry or non-symmetry of $A$, and in the assumptions on the form of the error. See Stewart and Sun (1990, Chap. IV and V) for a representative set of error bounds. Examples of concrete error bounds for structural similarities are given in Brandes et al. (2006) and Brandes and Lerner (2006). In some cases, looking for large gaps in the spectrum (thus maximizing stability) can also guide the analyst to determine the appropriate number of eigenvalues.

\section{Spectral Graph Partitioning}

In this section we briefly outline one possible application scenario for structural similarities that has been treated more thoroughly in Brandes and Lerner (2006). The problem we consider is the computation of graph colorings for random graphs that are drawn from certain probability distributions. A (proper) $k$-coloring of a graph $G=(V, E)$ is an assignment of $k$ colors to vertices, $c: V \rightarrow\{1, \ldots, k\}$, such that adjacent vertices are always colored differently. From a different point of view, a $k$-coloring is a special type of prespecified blockmodeling, where the vertices of a graph have to be partitioned into $k$ classes such that the diagonal blocks of the adjacency matrix are zero blocks and no restrictions are imposed on the off-diagonal blocks.

The problem of computing a $k$-coloring of a graph is NP-hard for any fixed $k \geq 3$, even if it is known that the graph is $k$-colorable (Garey and Johnson 1979). Nevertheless, there exist efficient heuristic algorithms that compute with high probability (i. e., with probability that tends to one 
when the number of vertices tends to infinity) a proper $k$-coloring on random graphs that are drawn from certain probability distributions.

A random graph model that has often been considered in this context (Alon and Kahale 1997; McSherry 2001) is outlined in the following. Let the matrix $A_{1}$ be defined as in (4) and assume that the rows and columns of $A_{1}$ are indexed by the vertices of a random graph.

$$
A_{1}=\left[\begin{array}{ccc|ccc|ccc}
0 & \cdots & 0 & p & \cdots & p & p & \cdots & p \\
\vdots & & \vdots & \vdots & & \vdots & \vdots & & \vdots \\
0 & \cdots & 0 & p & \cdots & p & p & \cdots & p \\
\hline p & \cdots & p & 0 & \cdots & 0 & p & \cdots & p \\
\vdots & & \vdots & \vdots & & \vdots & \vdots & & \vdots \\
p & \cdots & p & 0 & \cdots & 0 & p & \cdots & p \\
\hline p & \cdots & p & p & \cdots & p & 0 & \cdots & 0 \\
\vdots & & \vdots & \vdots & & \vdots & \vdots & & \vdots \\
p & \cdots & p & p & \cdots & p & 0 & \cdots & 0
\end{array}\right]
$$

To draw a sample from this random graph model include an edge $\{u, v\}$ independently from all other edges with probability equal to the $u v^{\prime}$ th entry of $A_{1}$ (i. e., with probability $p$ if $u$ is in a different color class than $v$ and with probability 0 else). A coloring algorithm gets such a random sample and has to recover the planted or latent 3-coloring. (Note that, of course, this algorithm does not know the particular vertex-order that led to the block-form of $A_{1}$.)

Traditional spectral algorithms (Alon and Kahale 1997; McSherry 2001) solve this problem by projecting the vertices to those three eigenvectors of the sampled adjacency matrix whose eigenvalues have the maximal absolute values and subsequently apply a clustering algorithm to obtain a discrete partition. However, this strategy works only for planted partition models where the blocks in the expected adjacency matrix are constant; it fails if we allow more general block-types, such as the blocks of an exact regular partition.

To generalize this model, let $X, Y$, and $Z$ be $r \times r$ matrices of probabilities (not necessarily constant) and consider the matrix $A_{2}$ in (5) which defines a distribution $\mathrm{G}\left(A_{2}\right)$ of 3-colorable graphs by including edges $\{u, v\}$ independently with probability equal to the $u v^{\prime}$ th entry of $A_{2}$.

$$
A_{2}=A[X Y Z]=\left[\begin{array}{ccc}
{[0]} & X & Y \\
X^{\mathrm{T}} & {[0]} & Z \\
Y^{\mathrm{T}} & Z^{\mathrm{T}} & {[0]}
\end{array}\right]
$$


A Generalized Spectral Algorithm. With the framework of structural similarities we can design a more general spectral algorithm that succeeds for more general choices of the $X, Y, Z$-in particular if these blocks are exact regular and satisfy an additional criterion. This algorithm is presented below and gets the $n \times n$ adjacency matrix $\hat{A}$ of a sampled graph as input.

\section{Spectral 3-Coloring Algorithm $(\hat{A})$}

1. Compute $d=\sum_{i, j=1}^{n} \hat{A}_{i j} /(2 n)$.

2. Compute (orthonormalized) eigenvectors $\left\{v_{1}, v_{2}, v_{3}\right\}$ of $\hat{A}$ associated to those eigenvalues that are the closest to $2 d,-d$ and $-d$.

3. Let $P$ be the $3 \times n$ matrix whose rows are the $v_{i}$ and compute $\hat{S}=P^{\mathrm{T}} P$.

4. Compute the pairwise distances of vertices according to the distance between their columns of $\hat{S}$.

5. Successively join vertices with the smallest distance until three classes are left.

6. Return the computed classes as a 3-coloring.

In Brandes and Lerner (2006) it has been shown that the coloring computed by the above algorithm is, with high probability, a proper coloring if the matrices $X, Y$, and $Z$ in (5) are regular of common degree $d$ and if the eigenvalues $2 d,-d$, and $-d$ of $A[X Y Z]$ are well-separated from the other eigenvalues. We let $\operatorname{sep}(A)$ denote the minimal distance from $2 d,-d$, and $-d$ to any other eigenvalue of $A$.

Theorem 6. (Brandes and Lerner (2006)) Let $X, Y$, and $Z$ be regular matrices of common degree $d$ and $A=A[X Y Z]$. Let $\sigma^{2}=\max _{u, v}\left(A_{u v}-A_{u v}^{2}\right)$ be the maximal variance of entries of $\mathrm{G}(A)$ and assume that $\operatorname{sep}(A)$ is in $\omega(n \sigma)$ and that $\sigma^{2} \gg\left(\log ^{6} n\right) / n$. Then, the Spectral 3-Coloring Algori thm properly 3-colors graphs from $\mathrm{G}(A)$, with high probability.

Sketch of Proof: It can be verified that if the blocks $X, Y$, and $Z$ are regular of degree $d$ then the partition into the 3 color-classes induces a structural similarity $S$ for the expected adjacency matrix, whose associated quotient is a triangle with edge weights equal to $d$. Further, we can show that this similarity is stable enough such that the sampled adjacency matrix admits a structural similarity $\hat{S}$ that is sufficiently close to $S$ such that it also reveals the planted color classes. Details are given by Brandes and Lerner (2006).

Insufficiency of Traditional Spectral Methods. It has been shown that the above algorithm indeed extends the application domain of spectral algorithms. 
Theorem 7. (Brandes and Lerner (2006)) For arbitrary large $r$, there are $r \times r$ matrices $X, Y$, and $Z$ such that graphs from $\mathrm{G}(A[X Y Z])$ are not colored properly by the algorithms in Alon and Kahale (1997) and McSherry (2001), but the Spectral 3-Coloring Algorithm succeeds on these graphs.

The proof of Theorem 7 goes by constructing graphs with a planted 3 -coloring in which large (i. e., of size linear in $n$ ) sets of differently colored vertices induce no edge. Details are given in Brandes and Lerner (2006).

Traditional spectral methods required constant blocks-compare the matrix $A_{1}$ in (4). The algorithm based on structural similarities works for the blocks of exact regular partitions. From the point of view of the coloring this means that vertices are no longer required to have the same probability to connect to all vertices of different color, but they are only required to have the same expected number of neighbors of each color different from their own.

Adaptation to Prespecified Blockmodeling. Note that, although we considered in this section the (rather specific) problem of 3-coloring graphs, the above results can be easily generalized to other tasks in prespecified blockmodeling, provided that the following assumptions are given. First, all blocks must be regular, i. e., for each block $B$ there are two numbers $r_{B}$ and $c_{B}$ such that all rows of $B$ sum up to $r_{B}$ and all columns of $B$ sum up to $c_{B}$ (in other words, the planted partition must correspond to an exact regular equivalence). Second, the eigenvalues of the quotient must be separated from the rest of the spectrum, such that the associated eigenvectors are stable with respect to random deviations. In comparison to our results, the optimizational approach to blockmodeling (Batagelj et al. 1992) is more general since it allows for other types of partitions (e. g., regular but not exact regular). On the other hand, we are not aware of results similar to Theorem 6 for local optimization algorithms, e.g., Batagelj et al. (1992).

\section{Visualization of Dynamic Conflict Networks}

The framework of structural similarities is especially convenient to develop methods for the analysis of large, noisy, empirical data sets. Brandes et al. (2006) applied structural similarities to visualize dynamic networks of conflict between political actors. We take this application scenario to illustrate the usage and usefulness of Theorem 3.

Conflict networks are networks where the edges have a negative or hostile interpretation, like criticism, accusations, or military engagements. Edges arise from time-stamped events between the actors involved. Given 
a conflict network, we generate a dynamic visualization that shows which group of actors is in opposition to which other group, which actors are most involved in conflict, and how do conflicts emerge, change their structure, and fade out over time. The example data sets are from the Kansas Event Data System (Schrodt, Davis, and Weddle 1994) and consist of approximately 78,000 dyadic events between political actors in the Balkans regions.

We make the assumption that actors are loosely grouped together such that conflicts occur mostly between members of different groups. Thus, an actor is a member of one out of $k$ classes to the extent that it has conflicts with members of the other classes. In the following, the method is described for the situation where there are only two groups that are mutually in conflict; an extension to more general conflict structures is given in Brandes and Lerner (2008).

To obtain a continuous assignment, we determine the degrees of membership of each actor to the two classes of the quotient $R_{c w}$ shown in Figure 5 (left). The eigenvalues of $R_{c w}$ are

$$
\lambda=c+w \quad \text { and } \quad \mu=c-w .
$$

From a different perspective the edge-weights of the quotient $R_{c w}$ are determined by its two eigenvalues $\lambda$ and $\mu$ as

$$
c=\frac{\lambda+\mu}{2} \quad \text { and } \quad w=\frac{\lambda-\mu}{2} .
$$

Theorem 3 implies that a similarity $S$ is structural with $G / S=R_{c w}$, if and only if $S$ is the projection onto the eigenvalues $\lambda$ and $\mu$ of $R_{c w}$. Since our goal here is to maximize the edge weight between the clusters, i. e., to maximize $w$, the optimal choice are the largest and the smallest eigenvalue of the adjacency matrix.

To obtain the actual degrees of membership to the two groups, the appropriate basis for the two-dimensional image space has to be identified. In short, the matrix $P$ whose rows are the two eigenvectors has to be rotated by the inverse eigenvector-basis of $R_{c w}$ (details can be found in Brandes et al. (2006)). An example for a projection to conflict space can be seen in Figure 5 (right).

To show the development over time, we defined in Brandes et al. (2006) time-dependent conflict networks that take into account only the events within a certain time-frame. By letting this time-frame move forward, we obtain a smoothly animated visualization. The smoothness of the animation, as well as the robustness with respect to noise in the input data, can be well characterized by a stability indicator called volatility (Brandes et al. 2006). It turns out that the projection is quite stable for all but a small 

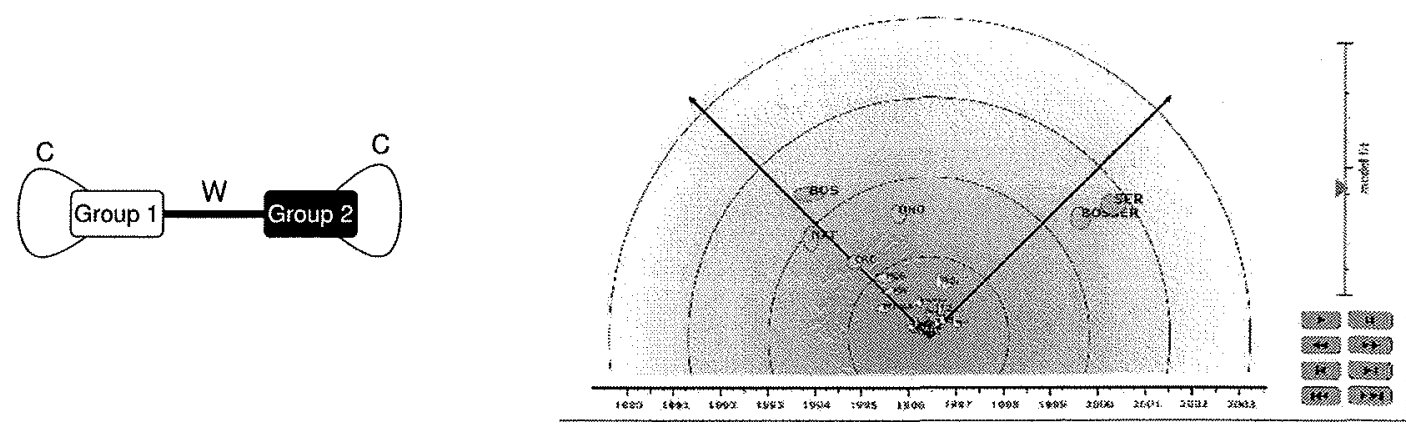

Figure 5. Left: Quotient $R_{c w}$ of a 2-dimensional conflict space; parameterized by the edge weights $c$ and w. Right: Conflictive groups in the Balkans for the period from 1989 until 2003. Actors are mapped into the left (right) dimension to the extent that they are members of one of the two groups. The distance from the origin is a measure of how involved actors are in conflict.

number of timepoint which, in turn, are characterized by a rapid change of the conflict structure.

Note that, in contrast to the application scenario outlined in Section 4 , the final result of the conflict network analysis is not a discrete partition but rather the continuous assignment of actors to the various groups. Advantages are that we can cope with actors that stand between groups (e. g., UNO in Figure 5) and that we can express varying involvement of actors (compare, e. g., BOS to CRO in Figure 5). Furthermore, a dynamic representation of the various groups, where the structure changes only slightly from a timepoint to the next, would not have been possible with discrete partitions. The computation of each picture is very fast, since only the two outer eigenvectors have to be computed; this is typically achieved by iterative algorithms that require linear time (in the number of vertices plus edges) in each step and have a geometric convergence rate (Golub and van Loan 1996).

\section{Conclusion}

In this paper we proposed the framework of structural similarities as a relaxation of the discrete notion of exact regular equivalence relations. Structural similarities are efficiently computable and their robustness with respect to noise is well characterized. The utility of structural similarities has been illustrated on two application scenarios. In Section 4 we showed that an algorithm based on the framework of structural similarities can provably recover latent vertex partitions from specific random graphs. In Section 5 we applied structural similarities to analyze and visualize dynamic networks of conflict among political actors. 
Structural similarities are an alternative to generalized blockmodeling (Doreian et al. 2005), if structural or exact regular blocks are sought. Furthermore, structural similarities lead to spectral graph partitioning methods that generalize traditional spectral graph analysis, see Section 3.2.2. Below we summarize how structural similarities compare to generalized blockmodeling and to traditional spectral methods.

Generalized Blockmodeling. Structural similarities are more restrictive with respect to ideal block types than generalized blockmodels. While structural similarities require (relaxed) blocks of structural or exact regular equivalences, generalized blockmodeling allows for other block types, such as row-regular, column-regular, row-functional or column-functional. On the other hand, structural similarities yield more efficient methods since they require only the computation of specific eigenvectors, which can be done efficiently for sparse networks. Furthermore, structural similarities are robust to perturbations of the input data, which enables them also to handle dynamically changing networks and yields provable guarantees for recovering latent vertex partitions in random networks.

Spectral Graph Analysis. Traditional spectral methods for graph analysis can recover latent vertex partitions if edge probabilities are only dependent on the class membership, i.e., in other words, if the latent partition is a perturbed version of a structural equivalence. Methods based on structural similarities generalize this to situations where the latent partition is a perturbed version of an exact regular equivalence. Thus the edge probabilities do no longer have to be constant within blocks; instead it is only required that vertices in one class have the same expected number of neighbors in the other class. Thus the range of spectral methods has been extended in a way that is needed in network analysis.

\section{References}

ACHLIOPTAS, D., FIAT, A., KARLIN, A., and MCSHERRY, F. (2001), "Web Search via Hub Synthesis", in Proceedings of the 42nd Annual IEEE Symposium on Foundations of Computer Science (FOCS'01), pp. 500-509.

ALON, N., and KAHALE, N. (1997), "A Spectral Technique for Coloring Random 3Colorable Graphs", SIAM Journal on Computation, 26, 1733-1748.

ARTIN, M. (1991), Algebra, Englewood Cliffs, NJ: Prentice Hall.

AZAR, Y., FIAT, A., KARLIN, A., MCSHERRY, F., and SAIA, J. (2001), "Spectral Analysis of Data", in Proceedings of the 33rd Annual ACM Symposium on Theory of Computing (STOC), pp. 619-626.

BAI, Z., DEMMEL, J., and MCKENNEY, A. (1993), "On Computing Condition Numbers for the Nonsymmetric Eigenproblem", ACM Transactions on Mathematical Software, 19, 202-223.

BATAGELJ, V. (1997), “Notes on Blockmodeling”, Social Networks, 19, 143-155. 
BATAGELJ, V., DOREIAN, P., and FERLIGOJ, A. (1992), "An Optimizational Approach to Regular Equivalence", Social Networks, 14, 121-135.

BORGATTI, S. P., and EVERETT, M. G. (1992), "Notions of Position in Social Network Analysis", Sociological Methodology, 22, 1-35.

BRANDES, U., and ERLEBACH, T. (eds.) (2005), Network Analysis: Methodological Foundations, Berlin, Heidelberg: Springer-Verlag.

BRANDES, U., FLEISCHER, D., and LERNER, J. (2006), "Summarizing Dynamic Bipolar Conflict Structures", IEEE Transactions on Visualization and Computer Graphics, special issue on Visual Analytics, 12, 1486-1499.

BRANDES, U., and LERNER, J. (2004), "Structural Similarity in Graphs", in Proceedings of the 15th International Symposium on Algorithms and Computation (ISAAC'04), pp. 184-195.

BRANDES, U., and LERNER, J. (2006), "Coloring Random 3-Colorable Graphs with NonUniform Edge Probabilities", in Proceedings of the 31 st International Symposium on Mathematical Foundations of Computer Science (MFCS '06), pp. 202-213.

BRANDES, U., and LERNER, J. (2008), "Visualization of Conflict Networks", in Building and Using Datasets on Armed Conflicts, ed. Kauffmann, M., IOS Press, vol. 36 of NATO Science for Peace and Security Series E: Human and Societal Dynamics, pp. 169-188.

COJA-OGHLAN, A. (2005), "A Spectral Heuristic for Bisecting Random Graphs", in Proceeding of the 16th ACM-SIAM Symposium on Discrete Algorithms, pp. 850-859.

CVETKOVIĆ, D. M., DOOB, M., and SACHS, H. (1995), Spectra of Graphs, HeidelbergLeipzig: Johann Ambrosius Barth.

DOREIAN, P., BATAGELJ, V., and FERLIGOJ, A. (2005), Generalized Blockmodeling, Cambridge, MA: Cambridge University Press.

EVERETT, M. G., and BORGATTI, S. P. (1991), "Role Colouring a Graph", Mathematical Social Sciences, 21, 183-188.

EVERETT, M. G., and BORGATTI, S. P. (1994), "Regular Equivalence: General Theory", Journal of Mathematical Sociology, 19, 29-52.

FIALA, J., and PAULUSMA, D. (2003), "The Computational Complexity of the Role Assignment Problem", in Proceedings of the 30th International Colloquium on Automata, Languages, and Programming (ICALP'03), Berlin Heidelberg: Springer-Verlag, pp. 817-828.

GAREY, M. R., and JOHNSON, D. S. (1979), Computers and Intractability: A Guide to the Theory of NP-completeness, San Francisco: Freeman and Company.

GKANTSIDIS, C., MIHAIL, M., and ZEGURA, E. (2003), "Spectral Analysis of Internet Topologies", in Proceedings of the IEEE INFOCOM'03, pp. 364- 374.

GODSIL, C. (1993), Algebraic Combinatorics, New York: Chapman \& Hall.

GODSIL, C., and ROYLE, G. (2001), Algebraic Graph Theory, New York: Springer.

GOHBERG, I., LANCASTER, P., and RODMAN, L. (1986), Invariant Subspaces of Matrices with Applications, New York: John Wiley.

GOLUB, G. H., and VAN LOAN, C. F. (1996), Matrix Computations, Baltimore, MD: John Hopkins University Press.

KAISER, H. F. (1958), "The Varimax Criterion for Analytic Rotation in Factor Analysis", Psychometrica, 23, 187-200.

KANNAN, R., VEMPALA, S., and VETTA, A. (2004), "On Clusterings: Good, Bad and Spectral", Journal of the ACM, 5I, 497-515. 
KIM, K. H., and ROUSH, F. W. (1984), "Group Relationships and Homomorphisms of Boolean Matrix Semigroups", Journal of Mathematical Psychology, 28, 448-452.

LORRAIN, F., and WHITE, H. C. (1971), "Structural Equivalence of Individuals in Social Networks", Journal of Mathematical Sociology, 1, 49-80.

LUCZKOVICH, J. J., BORGATTI, S. P., JOHNSON, J. C., and EVERETT, M. G. (2003), "Defining and Measuring Trophic Role Similarity in Food Webs Using Regular Equivalence", Journal of Theoretical Biology, 220, 303-321.

MCSHERRY, F. (2001), "Spectral Partitioning of Random Graphs", in Proceedings of the 42nd Annual IEEE Symposium on Foundations of Computer Science (FOCS'01), pp. 529-537.

MILNER, R. (1980), A Calculus of Communicating Systems, Berlin: Springer Verlag.

PAIGE, R., and TARJAN, R. E. (1987), "Three Partition Refinement Algorithms", SIAM Journal on Computing, 16, 973-983.

PAPADIMITRIOU, C., RAGHAVAN, P., TAMAKI, H., and VEMPALA, S. (2000), "Latent Semantic Indexing: A Probabilistic Analysis", Journal of Computer and System Sciences, 61, 217-235.

PATTISON, P. (1993), Algebraic Models for Social Networks, Cambridge, MA: Cambridge University Press.

SAILER, L. D. (1978), "Structural Equivalence: Meaning and Definition, Computation and Application", Social Networks, 1, 73-90.

SCHRODT, P. A., DAVIS, S. G., and WEDDLE, J. L. (1994), "Political Science: KEDS-A Program for the Machine Coding of Event Data", Social Science Computer Review, $12,561-588$.

STEWART, G. W., and SUN, J.-G. (1990), Matrix Perturbation Theory, New York: Academic Press.

VAN DE VELDEN, M., and KIERS, H. A. L. (2005), "Rotation in Correspondence Analysis", Journal of Classification, 22, 251-271.

VEMPALA, S., and WANG, G. (2002), "A Spectral Algorithm for Learning Mixtures of Distributions", in Proceedings of the 43rd Annual IEEE Symposium on Foundations of Computer Science (FOCS'O2).

WASSERMAN, S., and FAUST, K. (1994), Social Network Analysis: Methods and Applications, Cambridge MA: Cambridge University Press.

WHITE, D. R. and REITZ, K. P. (1983), "Graph and Semigroup Homomorphisms on Networks of Relations", Social Networks, 5, 193-234. 